\title{
Treatment of acute pancreatitis with pancreatic duct decompression via ERCP: A case report series
}

\author{
ZOUZHENG WANG ${ }^{*}$, QI WANG* ${ }^{*}$ JIANJUN SONG, MING LI, FENG WANG, \\ BENDONG CHEN, ZHENHUI LU, HONG CHEN, NA WANG, LI YONG and WEIJIE YAO \\ Department of Hepatobiliary Surgery, General Hospital of Ningxia Medical University, \\ Yinchuan, Ningxia Hui Autonomous Region 750004, P.R. China
}

Received November 19, 2019; Accepted February 4, 2020

DOI: $10.3892 /$ etm.2020.8962

\begin{abstract}
Acute pancreatitis is a common disorder in the gastrointestinal system, which is characterized by an increasing incidence and a high mortality. Currently, symptomatic treatment becomes the predominant option for the treatment of acute pancreatitis. To date, there is little knowledge on the treatment of acute pancreatitis through alleviation of pancreatic ductal hypertension and removal of pancreatic ductal obstruction. Endoscopic retrograde cholangiopancreatography (ERCP), an effective treatment for acute biliary pancreatitis, may alleviate the obstruction and edema in the common channel of the bile duct and pancreatic duct, to achieve the indirect treatment of acute pancreatitis, and may achieve the removal of intrapancreatic ductal obstruction and reduction in the intrapancreatic ductal pressure. Hereby, we report 3 cases with acute pancreatitis that were successfully treated by the pancreatic duct decompression via ERCP in one single center from China. Our data demonstrate that pancreatic duct decompression via ERCP is effective for the treatment of acute pancreatitis, which may shorten the course of acute pancreatitis through alleviating pain, shortening fasting duration and controlling the inflammatory reactions. It is recommended to use further prospective, randomized, controlled clinical trials to evaluate the efficacy and safety of pancreatic duct decompression via ERCP for acute pancreatitis.
\end{abstract}

\section{Introduction}

As a common disease in the gastrointestinal system, acute pancreatitis is characterized by increasing incidence and high

Correspondence to: $\mathrm{Dr}$ Zouzheng Wang, Department of Hepatobiliary Surgery, General Hospital of Ningxia Medical University, 804 Shengli South Street, Yinchuan, Ningxia Hui Autonomous Region 750004, P.R. China

E-mail: zt767d@163.com

Key words: acute pancreatitis, endoscopic retrograde cholangiopancreatography, pancreatic duct decompression, clinical efficacy mortality (1). Although there is timely update on the guidelines for the management of acute pancreatitis worldwide (2-4), symptomatic treatment currently remains the predominant option for the treatment of acute pancreatitis, including inflammation control, inhibition of pancreatic secretion, pain management, maintenance of stable blood circulation, and treatment of systemic or local complications (5).

Although multiple hypotheses have been proposed, the exact pathogenesis of acute pancreatitis remains unclear now. Multiple factors are considered to be involved in the pathophysiological process of acute pancreatitis, in which pancreatic ductal obstruction and hypertension may play critical roles (6). Endoscopic retrograde cholangiopancreatography (ERCP), an effective treatment for acute biliary pancreatitis, may alleviate the obstruction and edema in the common channel of the bile duct and pancreatic duct to achieve the indirect treatment of acute pancreatitis (7). To date, there is little knowledge on the treatment of acute pancreatitis through alleviation of pancreatic ductal hypertension and removal of pancreatic ductal obstruction. However, pancreatic ductal obstruction and hypertension is found in both biliary and non-biliary acute pancreatitis (8). Nevertheless, ERCP may be performed to remove intrapancreatic ductal obstruction and reduce intrapancreatic ductal pressure. Hereby, we summarize the experiences of successful treatment of acute pancreatitis through the pancreatic duct decompression via ERCP in one single center from China.

\section{Case reports}

Case 1. A 29-year-old woman was presented with complaints of intermittent right upper abdominal pain for 10 days. She was admitted to a local county hospital at the first onset of the pain, and was misdiagnosed as gastritis. However, the clinical symptoms did not relieve remarkably following acid suppressive and analgesic therapy. Three days ago, obvious aggravation of the middle and upper abdominal pain was found, complicated by fever. Elevated serum bilirubin and aminotransferase levels were detected in the local hospital, with serum amylase activity increased to 1,518 U/1. Transabdominal color Doppler ultrasonography displayed multiple multiple gallbladder stones, cholecystitis and pancreatic edema. The woman was then diagnosed as acute pancreatitis, and given conservative treatment. However, no satisfactory outcome was achieved, 


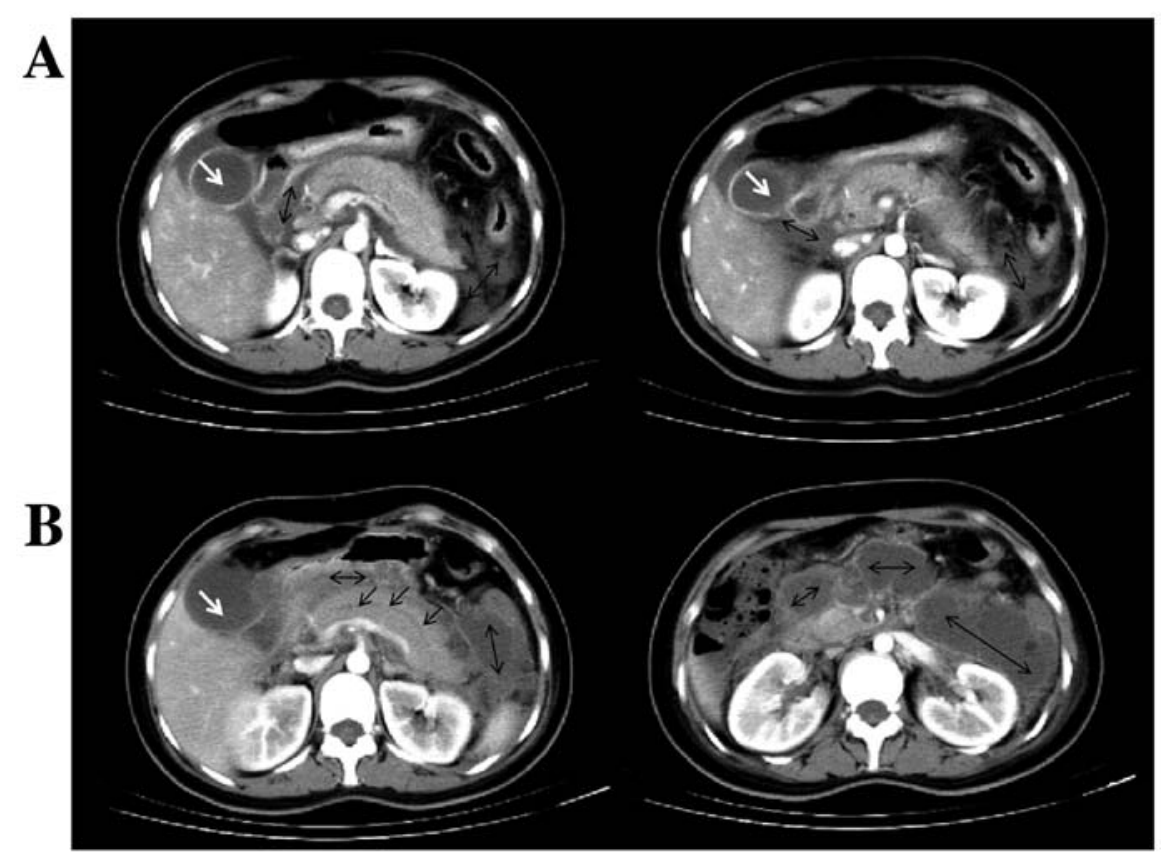

Figure 1. Preoperative imaging features of Case 1. (A) Abdominal enhanced CT scan displays gallbladder enlargement, a little effusion surrounding the gallbladder, remarkable pancreas swelling and patchy exudation shadows surrounding the pancreas at hospital admission; (B) following conservative therapy for 11 days, enhanced CT scan shows pancreatic duct dilatation, remarkable pancreatic parenchymal swelling with uniform and reduced density, and plenty of liquids gathering around the pancreas. White arrow represents the gallbladder; the black two-way arrow represents the peripancreatic fluid and the black one-way arrow represents the pancreatic duct.

and the case was then transferred to our hospital. She denied history of alcohol addiction or other medical history of specific diseases.

Admission physical examinations showed a body temperature of $38.9^{\circ} \mathrm{C}$, a heart rate of 125 beats $/ \mathrm{min}$; tenderness over the whole abdomen, notably in the middle and upper abdomen; and decreased bowel sounds. Blood testing revealed a WBC count of $25.4 \times 10^{9} / 1,50 \%$ hematocrit, $90.5 \%$ neutrophils, $1,499 \mathrm{U} / 1 \mathrm{LDH}, 360.9 \mathrm{U} / 1 \mathrm{AST}, 1,447 \mathrm{U} / 1$ amylase, $1.29 \mathrm{mmol} / 1$ $48 \mathrm{~h}$ serum calcium, and $3.3 \mathrm{mmol} / \mathrm{l}$ potassium. She had a Ranson score of 5 and an APACHE II score of 8. Abdominal enhanced CT scan revealed pancreatic parenchymal swelling, a little effusion surrounding the pancreas, and grade $\mathrm{C}$ acute pancreatitis according to the computed tomography severity index (Balthazar score) (Fig. 1A). Gallbladder stone and small terminal common bile duct stones were identified based on MRCP and transabdominal color Doppler ultrasonography; however, no respiratory or renal insufficiency was detected. Then, the woman was diagnosed as critical acute biliary pancreatitis.

The case was given conservative and symptomatic treatment, and underwent ERCP 11 days post-admission. Before treatment, she had a WBC count of $25.4 \times 10^{9} / 1,81 \%$ neutrophils, normal amylase, 1,092 U/l lipase, remarkable pancreatic parenchymal enlargement, a plenty of effusions around the pancreas and grade $\mathrm{E}$ acute pancreatitis at Balthazar score (Fig. 1B). During ERCP, a guidewire was inserted into the bile duct and cholangiography was performed. Filling defects were seen in the upper common bile duct, while truncation of the lower common bile duct was observed. Since the enlarged pancreatic head was considered to compress the common bile duct, endoscopic papillotomy (EPT) was performed. Another guidewire was inserted into the pancreatic duct, and a $5 \mathrm{Fr}$ pancreatic stent was placed along the guidewire. A lot of thick, purulent mucus was found coming out from the pancreatic duct (Video S1). Following insertion of nasobiliary tube along the biliary guidewire, purulent bile was found. Purulent pancreatic juice and bile were collected for microbiological examinations.

On day 1 after ERCP, the case had normal body temperature, a WBC count of $9.8 \times 10^{9} / 1,77.3 \%$ neutrophils, and alleviation of abdominal pain; however, turbid, purulent bile was seen in the nasobiliary tube, and mild tenderness was felt on the middle and upper abdomen. The woman had no abdominal pain or tenderness on her abdomen on day 2, with normal routine blood test and normal bile color. Bacterial culture of both pancreatic juice and bile showed Klebsiella pneumoniae infection. After discharge from hospital, she had no discomfort like abdominal pain or fever. Re-examinations showed normal serum and urine amylase activity and normal serum lipase 10 days after ERCP, and enhanced CT displayed a reduction of the pancreas size and multiple liquid accumulations around the pancreas, with grade $\mathrm{E}$ pancreatitis assessed (Fig. 2A). On day 14, unobstructed bile duct was found, and no filling defects were seen; and then, the nasobiliary tube was removed. On day 21 , an enhanced CT re-examination revealed that the pancreatic stent was in the place, the pancreas size and morphology returned to normal level, and no peri-pancreatic effusions were seen, which was classified as grade A pancreatitis (Fig. 2B). The case received selective cholecystectomy and pancreatic stent removal, and no onset of acute pancreatitis was reported at a long-term follow-up.

Case 2. A 33-year-old man was presented with complaints of persistent descending pain on his middle and upper abdominal pain for $8 \mathrm{~h}$, complicated by discontinuation of passage of 


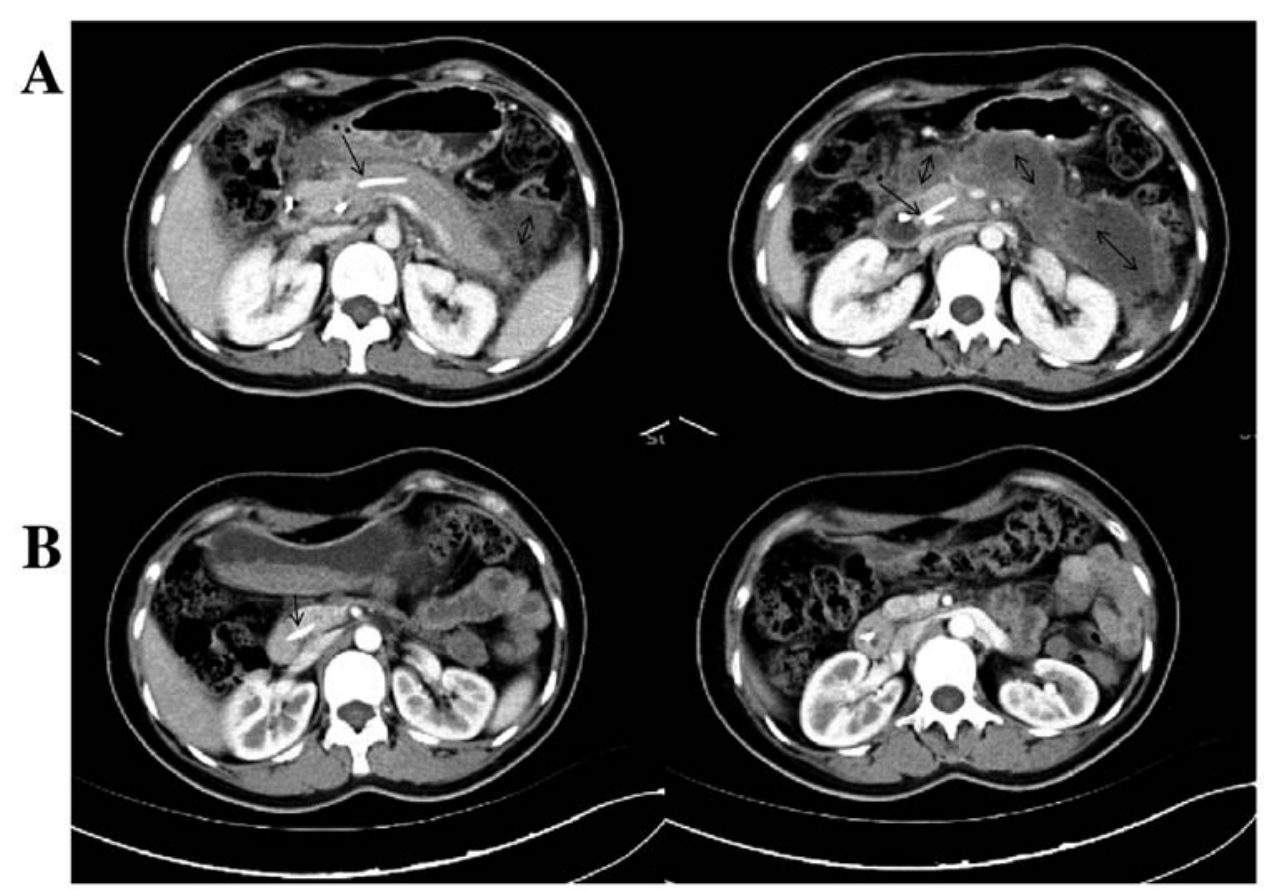

Figure 2. Preoperative imaging features of Case 1. (A) Enhanced CT scan shows a reduction in the pancreas volume, catheterization shadows in the pancreatic duct, and plenty of liquids gathering around the pancreas 10 days post-surgery; (B) Enhanced CT scan shows normal pancreas size and morphology, catheterization shadows in the pancreatic duct, and no remarkable effusion surrounding the pancreas 21 days post-surgery. The black two-way arrow represents the peripancreatic fluid and the black one-way arrow represents the pancreatic duct stent.

flatus and defecation. He had no fever, and denied history of alcohol addiction or other medical history of specific diseases. Admission physical examinations showed 26.37 BMI, abdominal fullness, tenderness and rebound pain over the upper abdomen, and bowel sounds were absent. Blood testing showed $17.3 \times 10^{9} / 1$ WBC count, $48.4 \%$ hematocrit, $92.7 \%$ neutrophils, 1,127 U/1 serum amylase, $604 \mathrm{U} / 1 \mathrm{LDH}, 49 \mu \mathrm{mol} / 1$ serum creatinine, and severe hyperlipidemia. He had a Ranson score of 3 and an APACHE II score of 3 . Abdominal ultrasonography indicated no gallbladder stone, and enhanced CT displayed remarkable pancreatic swelling and patchy exudations around the pancreas, and grade $\mathrm{C}$ pancreatitis (Fig. 3A). He was clinically diagnosed as moderately severe hyperlipidemic acute pancreatitis.

Following active conservative treatment, ERCP was performed. After the guidewire was inserted into the nipple, $\mathrm{X}$-ray scan showed the insertion of the guidewire into the pancreatic duct, and a lot of pancreatic juice and thick, purulent mucus came out from the pancreatic duct (Video S2). A 5 Fr pancreatic stent was placed along the guidewire, and the bile and pancreatic juice were collected for microbiological examinations. On day 1 after ERCP, the man had obvious relief from abdominal pain, distending pain on his back, mild tenderness over the middle and upper abdomen and peristalsis. Blood testing showed $9.52 \times 10^{9} / 1,80.3 \%$ neutrophils, and reduced but abnormal serum and urine amylase. On day 2 , he had no obvious abdominal pain or tenderness on the abdomen, normal serum and urine amylase and discharged from the hospital. Bacterial culture of both pancreatic juice and bile showed Escherichia coli infection. On day 7, enhanced CT re-examination displayed a reduction of the pancreas size, small necrotic foci in the pancreatic tail and liquid accumulations around the pancreas, with grade D pancreatitis identified (Fig. 3B). No specific interventions were given since the case had no comfort. One month after ERCP, an enhanced CT scan displayed normal pancreas size and morphology, no peri-pancreatic effusions, with grade A pancreatitis identified (Fig. 3C). The pancreatic stent was removed 2 months after ERCP, and no recurrence was found until April, 2017.

Case 3. A 39-year-old man was presented with complaints of the left upper abdominal pain complicated by nausea and vomiting for 7 days. He was diagnosed as acute pancreatitis in a local hospital based on abnormal elevation of serum and urine amylase levels. Following conservative therapy, the amylase activity restored to normal and abdominal pain relieved. However, abdominal pain developed again after the return to normal diet, and routine blood test showed $14.7 \times 10^{9} / 1$ WBC count. The man was then transferred to our hospital.

The case had no history of alcohol addiction. He suffered from gallbladder stone and acute cholangitis half a year ago, and was given laparoscopic cholecystectomy and ERCP. Admission physical examinations showed tenderness over the

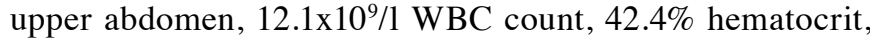
$69.5 \%$ neutrophils, $365 \mathrm{U} / 1 \mathrm{LDH}$, normal bilirubin and aminotransferase levels, mild elevation of serum amylase and lipase, and no manifestations of organ dysfunctions. He had a Ranson score of 1 and an APACHE II score of 1. Abdominal enhanced CT displayed pancreas tail enlargement and patchy exudations around the pancreas tail, and identified grade $\mathrm{C}$ pancreatitis. The man was therefore diagnosed as mild acute pancreatitis.

Following conservative treatment, including anti-infective therapy with third-generation cephalosporins, ERCP was performed. The duodenal papilla showed post-EPT alterations, and no filling defects were seen in the bile duct. Another guidewire was inserted into the pancreatic duct, and a pancreatic 


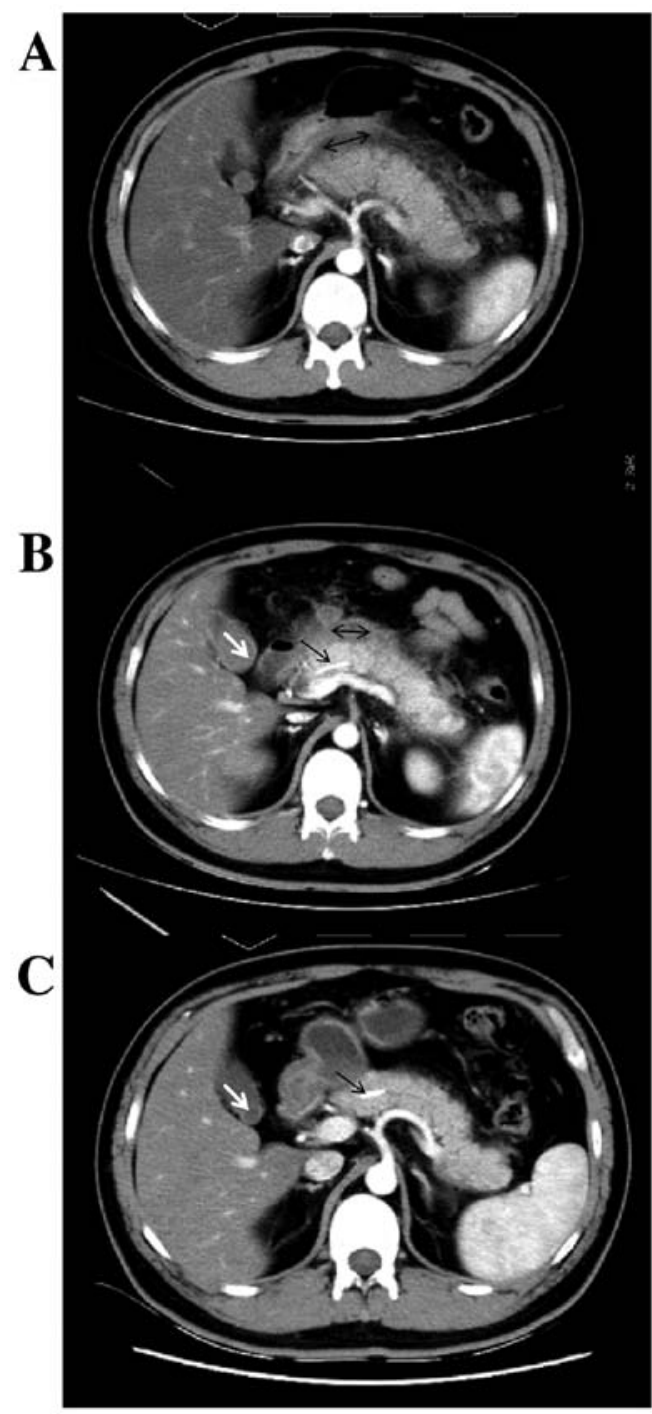

Figure 3. Preoperative imaging features of Case 2. (A) Enhanced CT scan displays uniform and reduced pancreas density, remarkable pancreatic parenchymal swelling and effusions surrounding the pancreatic parenchyma (B) Enhanced CT scan shows a reduction in the pancreas volume, dot-like necrotic foci at the pancreas tail and encapsulated effusion surrounding the pancreas 7 days post-surgery; (C) Enhanced CT scan shows normal pancreas size and morphology, catheterization shadows in the pancreatic duct, and no remarkable effusion surrounding the pancreas one month post-surgery. White arrow represents the gallbladder, the black two-way arrow represents the peripancreatic fluid, and the black one-way arrow represents the pancreatic duct stent.

stent was inserted along the guidewire, and detected flocculent, thick, purulent mucus. On day 1 after ERCP, abdominal pain was relieved, and normal diet was returned. After ERCP, the WBC count continued to be abnormal and showed an increase trend (with the greatest to $16.52 \times 10^{9} / 1$ ); however, the case had no fever. Bacterial culture revealed $E$. coli infection. Antibiotic susceptibility testing showed resistance to most cephalosporins, including all cephalosporins used for previous anti-infective therapy, and carbapenems were administered. Then, the WBC count reduced greatly, and returned to normal level after 5 day of carbapenems. The man had no rebound in the WBC count after the cease of anti-infective therapy, and was then discharged from the hospital. Pancreatic stent was removed 2 months after the discharge. During the follow-up period, the man had no abdominal pain, abnormal elevation of WBC counts or recurrence of acute pancreatitis.

\section{Discussion}

In this study, we presented the treatment of three cases with acute pancreatitis through removing pancreatic duct obstruction and insertion of pancreatic duct for decompression via ERCP. During ERCP, thick mucus was found to come out from the pancreatic duct. Following ERCP, abdominal pain was alleviated, and inflammation-related parameters reduced. Oral re-feeding was advanced after the procedure. Re-examinations showed complete absorption of peripancreatic effusions within one month after ERCP and recovery of pancreas morphology to normal. In addition, no recurrence of acute pancreatitis was found during the follow-up period.

To date, management of clinical symptoms and complications remains the predominant treatment for acute pancreatitis (5), since the pathogenesis of acute pancreatitis has not been fully demonstrated (1). Pancreatic ductal obstruction and hypertension has been considered as the primary cause of acute biliary pancreatitis (8); however, they also occur in non-biliary acute pancreatitis (9). In addition, it was reported that non-biliary acute pancreatitis was not completely dependent on bile duct obstruction and bile reflux (10). Therefore, bile duct obstruction and bile reflux are considered to be key events of acute pancreatitis (11). As an important approach in the diagnosis and treatment of pancreaticobiliary duct diseases, ERCP has been widely employed in pancreatic diseases. Notably, the effectiveness of ERCP for the treatment of biliary acute pancreatitis has been recognized throughout the world, and early ERCP is recommended for biliary acute pancreatitis, which has been proved to greatly reduce the morbidity and mortality of the complications of acute pancreatitis. However, ERCP is performed aiming to relieve the edema and obstruction in the common channel of the bile duct and pancreatic duct, thereby achieving the treatment of acute pancreatitis (7). Currently, there is little knowledge regarding the specific interventions targeting pancreatitis during ERCP. In our case series, thick, purulent mucus was found in the pancreatic duct during ERCP, suggesting both biliary and non-biliary acute pancreatitis is associated with pancreatic duct obstruction and hypertension. Similar findings have been obtained in animal studies in 1990s (10), and pancreatic duct hypertension was reported to positively correlate with the severity of acute pancreatitis(12), and may cause pancreatic ischemia and necrosis and aggravate pancreatitis $(13,14)$. In addition, the thick, purulent mucus may aggravate pancreatic duct hypertension, resulting in the aggravation of the disease and extension of the disease course. After the removal of the obstruction, normal pancreatic juice came out from the pancreatic duct, while insertion of a pancreatic stent may smooth the pancreatic juice drainage and relieve pancreatic duct hypertension, which may prevent pancreatitis recurrence caused by pancreatic duct obstruction again. At the same time, pancreatic duct decompression brought about obvious improve of inflammatory response. Inflammation indexes such as blood leukocytes and temperature, which was not well controlled by conservative treatment, improved significantly after ERCP. 
Pain is the most common initial symptom of acute pancreatitis, which may cause anxiety and even respiratory distress (15). Pain occurs across the entire course of acute pancreatitis, and is an important factor for the return to normal diet (2). Therefore, pain control is an important part of acute pancreatitis treatment. Analgesics are widely recommended to relieve the pain due to acute pancreatitis; however, opioids, anaesthetics and non-steroidal antiinflammatory drugs (NSAIDs) suffer from two problems of drug-related adverse reactions and repeated administration. Pancreatic duct hypertension has been proved to be the primary cause of chronic pancreatitis pain, and therefore, pancreatic duct decompression via ERCP is a common approach for the treatment of chronic pancreatitis (16). In acute pancreatitis patients, pancreatic duct decompression via ERCP may remarkably alleviate abdominal pain as well. In our case series, all three cases had obvious abdominal pain prior to ERCP, which required analgesics to alleviate pain, and the pain was effectively alleviated after ERCP without analgesics. All cases had their abdominal pain relief and their normal diet returned 1 to 3 days after ERCP. For instance, case 3 had normal serum amylase and alleviation of abdominal pain 6 days after conservative therapy; however, abdominal pain occurred again after eating. On day 1 after ERCP, he got pain relief and oral re-feeding. No abdominal pain was seen in all three cases after re-feeding, suggesting that abdominal pain correlates with pancreatic duct hypertension in acute pancreatitis patients, and pancreatic duct via ERCP may achieve an analgesic efficacy. Early return to normal diet facilitates the recovery of gastrointestinal functions and prevents the development of many complications, therefore shortens the course of acute pancreatitis.

Peripancreatic fluid accumulation is the most common local complication of acute pancreatitis. The imaging findings of pancreas cannot indicate the severity of acute pancreatitis. However, if peripancreatic exudations cannot be absorbed timely, these exudations may wall-off and even develop to peripancreatic infection or pseudocysts, resulting in aggravation of the disease and extension of the disease course. The outcome of local complications is therefore an important parameter that requires periodical monitoring during the treatment of acute pancreatitis. In our case series, peripancreatic exudations were completely absorbed following one-month after pancreatic decompression via ERCP, and the pancreas morphology significantly improved regardless of the amount of peripancreatic exudations or pancreatic parenchymal necrosis. It is recommended to wait for the peripancreatic fluid to be wrapped before performing interventions, such as percutaneous drainage, endoscopic drainage or surgical treatment (2-4). However, the intention of endoscopic pancreatic duct decompression and other interventions was not completely the same. Hypertension of the pancreatic duct can lead to increased pancreatic duct permeability and even rupture $(9,17)$, which was the reason why peripancreatic fluid was frequently rich in amylase. After ERCP dredged the pancreatic duct and thus reduced further overflow from pancreas, the effusion would also accelerate its own absorption. The second purpose was to directly drain the pancreatic or peripancreatic fluid for certain patients. After pancreatic decompression, other invasive interventions may be avoided. In addition, collecting pancreatic juice for bacterial culture can guide the application of antibacterial drugs, as shown in case 3. It is recommended to use further clinical application of pancreatic decompression via ERCP as the first choice to treat pancreatitis.

\section{Acknowledgements}

Many thanks are expressed to the three patients and their family members for their participation and active cooperation in this study.

\section{Funding}

This study was supported by the Ningxia Hui Autonomous Region Science and Technology Pillar Program (grant no. 2015KJHM40).

\section{Availability of data and materials}

The datasets used and/or analyzed during the current study are available from the corresponding author on reasonable request.

\section{Authors' contributions}

ZW and QW wrote the manuscript. JS and ML conceived and designed the study. $\mathrm{FW}$ and $\mathrm{BC}$ were responsible for the collection and analysis of the experimental data. ZL and $\mathrm{HC}$ interpreted the data and drafted the manuscript. NW, WY and LY revised the manuscript critically for important intellectual content. All authors read and approved the final manuscript.

\section{Ethics approval and consent to participate}

The study was approved by the Ethics Committee of General Hospital of Ningxia Medical University (Ningxia, China). Patients who participated in this research, signed the informed consent and had complete clinical data.

\section{Patient consent for publication}

Not applicable.

\section{Conflict of interest}

The authors declare that they have no competing interests.

\section{References}

1. Lankisch PG, Apte M and Banks PA: Acute pancreatitis. Lancet 386: 85-96, 2015.

2. Yokoe M, Takada T, Mayumi T, Yoshida M, Isaji S, Wada K, Itoi T, Sata N, Gabata T and Igarashi H, et al: Japanese guidelines for the management of acute pancreatitis: Japanese Guidelines 2015. J Hepatobiliary Pancreat Sci 22: 405-432, 2015.

3. Working Group IAP/APA Acute Pancreatitis Guidelines: IAP/ APA evidence-based guidelines for the management of acute pancreatitis. Pancreatology 13 (Suppl 2): e1-e15, 2013.

4. Tenner S, Baillie J, DeWitt J, Vege SS; American College of Gastroenterology. American College of Gastroenterology Guideline: Management of acute pancreatitis. Am J Gastroenterol 108: 1400-15, 2013.

5. Janisch NH and Gardner TB: Advances in management of acute pancreatitis. Gastroenterol Clin North Am 45: 1-8, 2016.

6. Bhatia M, Wong FL, Cao Y, Lau HY, Huang J, Puneet P and Chevali L: Pathophysiology of acute pancreatitis. Pancreatology 5: 132-144, 2005. 
7. Kapetanos DJ: ERCP in acute biliary pancreatitis. World J Gastrointest Endosc 2: 25-28, 2010.

8. Delhaye M, Matos C, Arvanitakis M and Deviere J: Pancreatic ductal system obstruction and acute recurrent pancreatitis. World J Gastroenterol 14: 1027-1033, 2008.

9. Harvey MH, Wedgwood KR, Austin JA and Reber HA Pancreatic duct pressure, duct permeability and acute pancreatitis. Br J Surg 76: 859-862, 1989.

10. Arendt T, Nizze H, Mönig H, Kloehn S, Stüber E and Fölsch UR Biliary pancreatic reflux-induced acute pancreatitis-myth or possibility? Eur J Gastroenterol Hepatol 11: 329-335, 1999.

11. Siqin D, Wang C, Zhou Z and Li Y: The key event of acute pancreatitis: Pancreatic duct obstruction and bile reflux, not a single one can be omitted. Med Hypotheses 72: 589-591, 2009.

12. Fujiwara H: Pressure measurement in pancreatic duct and biliary duct system in dogs with acute pancreatitis. Kobe J Med Sci 37: 47-55, 1991.

13. Shi CX, Chen JW, Carati CJ, Schloithe AC, Toouli J and Saccone GT: Effects of acute pancreatic duct obstruction on pancreatic perfusion: Implication of acute pancreatic duct decompression. Scand J Gastroenterol 37: 1328-1333, 2002.
14. Arendt T: Bile-induced acute pancreatitis in cats. Roles of bile, bacteria, and pancreatic duct pressure. Dig Dis Sci 38: 39-44, 1993.

15. Fantini L, Tomassetti P and Pezzilli R: Management of acute pancreatitis: Current knowledge and future perspectives. World J Emerg Surg 1: 16, 2006.

16. Seicean A and Vultur S: Endoscopic therapy in chronic pancreatitis: Current perspectives. Clin Exp Gastroenterol 8: 1-11, 2014

17. Nadkarni NA, Kotwal V, Sarr MG and Swaroop Vege S: Disconnected pancreatic duct syndrome: Endoscopic stent or surgeon's knife? Pancreas 44: 16-22, 2015.

This work is licensed under a Creative Commons Attribution-NonCommercial-NoDerivatives 4.0 International (CC BY-NC-ND 4.0) License. 